\title{
Warum werden wir eigentlich immer älter?
}

Es ist schon paradox: Die Lebenserwartung nimmt weiter zu und dies,

- obwohl kaum jemand die lebensdiätetischen Empfehlungen umsetzt,

- obwohl Kinder und Jugendliche immer dicker werden

- obwohl die Häufigkeit von Diabetes, Hypertonie und Dyslipidämie ständig zunimmt,

- obwohl viele Patienten die verordneten Medikamente gar nicht einnehmen,

- obwohl nur ganz Wenige die empfohlenen Früherkennungsuntersuchungen wahrnehmen,

- obwohl die meisten Ärzte ihre Patienten nicht leitlinienkonform behandeln,

- obwohl immer mehr Ärzte wegen ver- meintlicher Behandlungsfehler verklagt werden,

- obwohl bei immer mehr Patienten gravierende Nebenwirkungen der Medikamente auftreten,

- obwohl heute keiner mehr gesund, sondern nur unzureichend untersucht ist,

- obwohl durch die Unterfinanzierung des Gesundheitssystems viele Patienten unterversorgt sind,

- obwohl, obwohl, obwohl!

Doch wem haben wir diese erfreuliche Entwicklung eigentlich zu verdanken? Als Erstes meldeten sich die Kardiologen - selbstbewusst wie immer - und behaupteten frech, die längere Lebensdauer sei in erster Linie das Ergebnis ihrer Bemühungen, der
Beitrag der Onkologen dagegen sei nur marginal. Die anderen ärztlichen Berufsgruppen wurden zwar nicht explizit erwähnt, aber man darf wohl annehmen, dass innen auch keine besondere lebensverlängernde Wirkung zugestanden wird. Aber es muss einen doch stutzig machen, dass gerade der Bereich der Medizin, nämlich die Kardiologie, wo die Diskrepanz zwischen Anspruch und Wirklichkeit am größten sein soll, so segensreich wirken kann. Es ist ja gar nicht vorstellbar, was passieren würde, wenn alle das täten, was die Kardiologen uns empfehlen. Würden wir dann unsterblich?

Dr. med. Peter Stiefelhagen -
PROBLEMATISCHE ERNÄHRUNGSGEWOHNHEITEN

\section{Kein Sitzfleisch beim Essen}

— Im Alltag wird eine ausgewogene und gesunde Ernährung zunehmend zu einer Herausforderung, so das Ergebnis der Nestlé Studie 2011. Ein Großteil der Deutschen ernährt sich von Snacks und Zwischenmahlzeiten. Zeit und Ruhe für das Essen fehlen. Für die neue Ernährungsstudie wurden über 4000 Bundesbürger befragt. Immer öfter essen die Menschen aus Frust und Stress (+ 5\% seit 2009), obwohl 67\% der Deutschen beim Essen Zeit und Ruhe sogar für wichtiger halten als Abwechslung, Frische oder Gemeinsamkeit. Der Hauptgrund dafür ist der Studie zufolge der immer stärker von Flexibilität und Mobilität bestimmte Alltag vieler Bundesbürger. Jeder dritte Befragte ist ständig oder mindestens teilweise einem unregelmäßigen Tagesablauf unterworfen, mit steigender Tendenz. In der Folge nehmen sich nur noch 53\% der Studienteilnehmer die Zeit, um in Ruhe essen zu können, 45\% fehlt dafür die Zeit.

Die weitaus meisten Vollzeit-Berufstätigen (80\%) essen mittags außer Haus. Gerade Jüngere ersetzen immer öfter Hauptmahlzeiten durch Snacks. Sie essen zu spät, zu ungesund, zu unregelmäßig und mit Heißhunger. Rund 40\% nehmen zu viel Süßes und zu viel Fastfood zu sich. In der Studie wird folgende Konsequenz gezogen: „Der Trend zur spontanen, unstrukturierten Ernährung erfordert zum einen eine wesentlich konsequentere Ernährungserziehung von Kindern und Jugendlichen, zum anderen eine Optimierung des Snacking-Angebots, um zu verhindern, dass die zunehmende Spontaneität zu Ernährungsdefiziten führt."

$J M$.

DLO, Nestlé Deutschland,18. Januar 2011, www.nestle-studie.de
FRÜHER UMSTIEG AUF FESTE KOST

\section{Flaschenkinder werden so eher dick}

— Ein sechsfach erhöhtes Adipositasrisiko haben Kleinkinder, die als Babys zu früh feste Nahrung bekamen; allerdings nur dann, wenn sie nie oder weniger als vier Monate lang gestillt wurden. Die

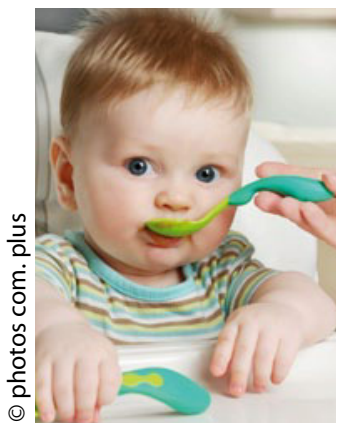

Zu früh mit dem Brei begonnen? Autoren haben Daten von 847 Kindern aus dem "Project Viva“ ausgewertet, einer Studie über mindestens drei Jahre. $67 \%$ der Kinder hatten die Brust bekommen, 32\% das Fläschchen. Im Alter von drei Jahren waren 9\% übergewichtig. Bei den Fläschchenkindern, die vor Ablauf von vier Monaten auf Brei umgestiegen waren, war die Wahrscheinlichkeit, mit drei Jahren übergewichtig zu werden, 6,3-fach erhöht. Diese Assoziation bestand bei vorher ausschließlich gestillten Kindern nicht. Studienautorin Huh mutmaßt, dass stillende Mütter zuverlässiger merken, wann ihr Kind satt ist, während die Mütter von Fläschchenkindern deren Nahrungsbedarf möglicherweise nicht so gut einschätzen können. EO = Huh S. Y. et al., Pediatrics online, 7. Februar 2011 